\title{
Examples of groups in abstract Algebra Course Books
}

\author{
Fulya Kula ${ }^{1 a}$ \\ ${ }^{1}$ Amasya University, 05100, Amasya, Turkey
}

\begin{abstract}
This study has been conducted with the aim to examine the examples of Abelian and non-Abelian groups given in the abstract algebra course books in the university level. The non-examples of Abelian groups serve as examples of non-Abelian groups. Examples with solutions in the course books are trusted by the students and hence miscellaneous of those are required to clarify the subject in enough detail. The results of the current study show that the examples of Abelian groups are about the same among three course books, including number sets only with known operations. The examples of non-Abelian groups are rare in comparison and encapsulate the nonnumeric sets which are novel to students. The current study shows the mentioned examples are not sufficiently examined in the course books. Suggestions for the book writers are given in the study. Mainly it is suggested that more and various examples of Abelian and especially non-Abelian groups should be included in the course books.
\end{abstract}

Keywords: abstract algebra; abelian groups; examples; course books

\section{Introduction}

Algebra is a broad section of mathematics and abstract algebra is one of algebra's sub-areas which studies algebraic structures singly. In abstract algebra, a group is a set of elements defined with an operation that integrates any two of its elements to form a third element satisfying four axioms. These axioms to be satisfied by a group together with the operation are; closure, associativity, identity and invertibility and are called group axioms. The integers as a number set together with the addition operation is a familiar example of a group and denoted with $(\mathrm{Z},+)$. The ubiquity of groups within and outside mathematics assigns them a central role in the organizing principle of contemporary mathematics. Group theory is not an untouchable, pure subject without applications. Some applications of groups in real world can be summarized as the study of crystals, Rubik's cube, the coding theory and error correcting codes, chemical areas, robotics, and medical image analysis.

\footnotetext{
${ }^{a}$ Corresponding author: fulya.kula@amasya.edu.tr
} 
Abelian groups also called as commutative groups, are groups which satisfy the axiom of commutativity, namely the result of applying the group operation to any two elements does not depend on their order. Abelian groups are named after Niels Henrik Abel and are one of the first and basic concepts of undergraduate abstract algebra. Hence Abelian groups satisfy all of the five the axioms: closure, associativity, identity, invertibility and commutativity. Besides, a group which is not commutative is called a "non-abelian group" or "non-commutative group".

Abelian groups are generally perceived simpler than non-abelian ones. Finite abelian groups are very well understood mainly because of the ease of the demonstration of axioms to finite number of elements of the set. On the other hand, the theory of infinite abelian groups is in the scope of the current research. A simple and common example of an Abelian group is the set of integers together with the operation addition "+". The addition operation, performed with integers, combines any two integers and forms a third integer (closure), is associative, zero is the additive identity (identity), all integers have an additive inverse which is the negative of that integer (invertibility). These axioms confirm that $(Z,+)$ is a group. Moreover, the addition operation is commutative under integers as $m+n=n+m$ for any two integers $\mathrm{m}$ and $\mathrm{n}$ (commutativity). In most books and courses of abstract algebra, $(\mathrm{Z},+)$ is common and first example used.

The non-abelian or noncommutative groups are groups for which the commutativity axiom does not hold, i.e. for arbitrary two elements $m$ and $n$ of the group $(G, *)$ the equation $m^{*} n=n * m$ does not hold. One of the most common non-abelian group is the dihedral group of order 6, which is a finite non-abelian group. An example of non-algebraic groups is the rotation group $\mathrm{SO}(3)$ in three dimensions, in physics discipline which means that when one rotates anything 90 degrees away from himself and then 90 degrees to the left is not the same as doing this the other way round. Non-abelian groups are used also in gauge theory in physics. Hence non-abelian groups also have various applications. The Abelian groups are easier to perceive and show than non-Abelian ones [7]. Therefore for the clarification of a non-abelian group, the non-examples should be built and the attention of students should be taken, both by the teacher and by the course books. While the concept of groups is an integral part of abstract algebra, studies of instruction of groups are found to be rare.

In this respect the significance of examples appear as the most important tools in education with a main place in most theories of learning mathematics [3, 6]. Examples are key when developing conceptual understanding of mathematical ideas, they give insight to mathematical concepts' definitions, theorems, and proofs $[4,5]$. By the power of examples in pedagogical environments $[3,6]$, researchers shape students' ideas and knowledge of mathematical concepts [1]. The broad categories of examples are in three main labels: 'generic example', 'counter-example' and 'non-example' [2]. Generic examples are either examples of concepts and procedures, or constitute the core of a generic 'proof'. Counterexamples disprove a hypothesis or assertion. Non-examples serve to make the boundaries clear where a procedure may not be applied or fails to produce the desired result. The examples for non-Abelian groups are included to the non-examples of Abelian groups. While there are available studies of examples in the literature the current study is limited to the examples and nonexamples.

Students generally work with number sets with the four operations throughout their academic lives untill the undergraduate level. The algebra and abstract algebra courses encountered in the university levels give a different point of view about these sets and operations to form algebraic groups or rings. Hence it is conceivable that students think of these number sets and four operations as references to algebraic structures. This fact may be a reason for the undergraduate students to fail to notice some algebraic properties 
thoroughly. In this respect, the non-examples, encountered by the course books or by the instructors are important to disprove the proposition that all groups are Abelian.

The idea of the current study was rooted in abstract algebra courses and students' questions regarding the overgeneralization of groups to number groups, and, as a result, their wrong conclusions that all groups have to be Abelian. The aim of this study is to explore and analyze the examples of groups, specifically Abelian and non-Abelian groups in the abstract algebra course books.

\section{Method}

The content analysis method in the qualitative data analysis was carried out to analyze the data of the current study in stages of data coding, categorizing, identification and interpretation. Content analysis is a method of analysing and summarizing any written text such as articles, books or book chapters, papers, letters and historical articles with certain rules [8].

\subsection{The context and process of the study}

In this study, it is aimed to determine the examples and non-examples in abstract algebra books. The first criteria to select and review the books to be analyzed in the study were determined as to be an abstract algebra book, include the chapter of groups, with also Abelian and non-Abelian groups. The purposeful sampling was used for the selection of the books. All the books examined were written in English language. the books were published in 1995, 2002 and 2005. The printed versions of the books were gathered from a university library in Turkey. The examples examined in these books were those which was presented duing the cover of the topic, hence worked examples are included in the current study while exercises and to be solved questions without solutions or explanations are not included. Each item which is the example of an Abelian or non-Abelian group was numbered and saved apart from the course book by two researchers independently.

\subsection{Analysis of data}

In total 25 examples were examined. The items were analyzed by two researchers independently. The data were analyzed descriptively by converting to tables and frequency tables.

\section{Results}

The examples were grouped according to whether or not it is an Abelian group example. The frequencies of examples for Abelian groups and non-Abelian groups are presented in Table 1.

Table 1. Frequencies of examples in course books.

\begin{tabular}{llll}
\hline Frequency & Course Book 1 & Course Book 2 & Course Book 3 \\
\hline Abelian group examples & 6 & 7 & 5 \\
Non-Abeian group examples & 2 & 3 & 2 \\
Total & 8 & 10 & 7 \\
\hline
\end{tabular}


As shown in Table 1, many examples are given for the Abelian groups in the course books. It can further be seen that the number of non-Abelian groups are limited.

Table 2. Examples in the course books.

\begin{tabular}{|c|c|c|c|}
\hline Frequency & Course Book 1 & Course Book 2 & Course Book 3 \\
\hline $\begin{array}{l}\text { Abelian group } \\
\text { examples }\end{array}$ & $\begin{array}{l}(\mathrm{Z},+),(\mathrm{Q},+),(\mathrm{R},+), \\
(\mathrm{Q}+, .),(\mathrm{R}+, .), \\
\text { Every cyclic group } \\
\mathrm{G}\end{array}$ & $\begin{array}{l}(\mathrm{Z},+),(\mathrm{Q},+),(\mathrm{R},+), \\
(\mathrm{C},+),\left(\mathrm{Q}^{*}, .\right),\left(\mathrm{R}^{*}, .\right), \\
\left(\mathrm{C}^{*}, .\right)\end{array}$ & $\begin{array}{l}(\mathrm{Z},+),(\mathrm{Q},+) \\
(\mathrm{R},+),(\mathrm{Q}+, .),(\mathrm{R}+, .)\end{array}$ \\
\hline $\begin{array}{l}\text { Non-Abeian group } \\
\text { examples }\end{array}$ & $\begin{array}{l}\text { The group of non } \\
\text { singular matrices, } \\
\text { GL(n)=\{A } \\
\square \mathrm{M}(\mathrm{n}): \operatorname{det}(\mathrm{A}) \neq 0\}, \\
(\mathrm{GL}(\mathrm{n}), .)\end{array}$ & $\begin{array}{l}\text { The set of all nxn } \\
\text { matrices with real } \\
\text { numbers as entries } \\
\text { M(n), Sn for all } n>=3 \\
\text { The dihedral group of } \\
\text { order 6, D3 }\end{array}$ & $\begin{array}{l}\text { Symmetric } \\
\text { group } \mathrm{S} 3 \text { of order } 6 \text {, } \\
\text { Rotation group } \\
\mathrm{SO}(3)\end{array}$ \\
\hline
\end{tabular}

The examples of groups that are Abelian or non-Abelian are examined and illustrated above in Table 2. The findings of the study in Table 2 point that, Abelian group examples generally took part with only number sets. On the other hand, all the non-examples or nonAbelian examples use sets which are encountered recently. The non-Abelian examples are found to be poorly explained, that is not enough detail in each item was considered to prove that the property of commutativity does not hold.

\section{Discussion and conclusion}

The Abelian groups are easier to perceive with regard to their counterparts as also perceived in the applications of non-Abelian groups [7]. Hence the non-Abelian group examples should be given with enough detail in the instruction. The students are familiar with number sets mostly and they may have the tendency to overgeneralize the structure of these sets. Consequently the distinction of the Abelian and non-Abelian groups is essential. For this reason the non-examples need to be covered with enough detail to clarify the difference between the two groups. Examining three abstract algebra course books this study shows that the examples of Abelian groups are much more than of the non-Abelian ones. When the examples in the course books are considered individually, it is determined that the Abelian group examples mostly took part with well-known number sets and operations. Examples of non-Abelian groups are distinctly fewer. While it is an expected outcome not to include number sets in the course books, the very few examples with nonnumeric sets for Abelian groups is worth considering. On the other hand, the nonAbelian group examples were not discussed in enough detail in the course books. This is thought to be resulted from the early consideration of the said nonnumeric set in the course book. However such nonnumeric set examples may be novel to students. Hence it is thought that these non-examples should be clarified by necessarily showing the property of commutativity does not hold. It is suggested within this study that the course book writers include examples of nonnumeric sets for Abelian groups like it is the case in non-Abelian group. Moreover examples for non-Abelian groups in course books need to be increased not only in quantity but also in quality. The detailed presentation that the commutative property is not valid for the examples of non-Abelian groups is also among the suggestions of the current research. It is recommended that the examples of the instructors during the courses also should be examined in the future research. Together with the suggested 
research, students' conceptions in Abelian and non-Abelian groups may be considered in detail to raise a component of the studies of misconceptions in abstract algebra.

\section{References}

1. Alcock, L. J. Mathematicians' perspectives on the teaching and learning of proof. Research in Collegiate Mathematics Education VII, 63-92, (2010).

2. Bills, L., Dreyfus, T., Mason, J., Tsamir, P., Watson, A., \& Zaslavsky, O. Exemplification in mathematics education. Proceeding of the 30th Conference of the International Group for the Psychology of Mathematics Education. Prague, Czech Republic: PME, (2006).

3. Bills, E. \& Watson, A. (Eds), Special issue: The role and use of examples in mathematics education. Educational Studies in Mathematics 69, 77-79, (2008).

4. Cuoco, A., Goldenberg, E. P., \& Mark, J. Habits of mind: An organizing principle for mathematics curriculum. Journal of Mathematical Behavior, 15(4), 375-402, (1997).

5. Lakatos, I. Proofs and Refutations: The Logic of Mathematical Discovery. Cambridge: Cambridge University Press, (1976).

6. Mason, J. \& Watson, A. Mathematics as a Constructive Activity: exploiting dimensions of possible variation. In M. Carlson \& C. Rasmussen (Eds.) Making the Connection: Research and Practice in Undergraduate Mathematics, Washington: MAA. 189-202, (2008).

7. Paeng, S. -H., Ha, K.-C., Kim, J. H., Chee, S. \& Park, C. New Public Key Cryptosystem Using Finite non Abelian Groups. In Kilian, J. (Ed.) Advances in Cryptology - CRYPTO. LNCS, vol. 2139, Springer, Heidelberg, 470-485, ( 2001).

8. Patton, M.Q. Qualitative Research. Encyclopedia of Statistics in Behavioral Science. New Jersey: John Wiley \& Sons, (2005). 